\title{
Transient receptor potential channels in multiple myeloma (Review)
}

\author{
LINGJUN MENG* ${ }^{*}$, GUIYING GU* and LINTAO BI \\ Department of Hematology and Oncology, China-Japan Union Hospital of Jilin University, \\ Changchun, Jilin 130033, P.R. China
}

Received November 20, 2021; Accepted December 30, 2021

DOI: $10.3892 / \mathrm{ol} .2022 .13228$

\begin{abstract}
Multiple myeloma is the second most commonly diagnosed hematologic malignancy. As an incurable disease, the molecular mechanisms underlying its many aspects remain unclear. Intracellular calcium ion is an essential signaling molecule that modulates malignant cell behavior, and abnormal regulation of cellular calcium homeostasis may promote cancer cell survival and induce drug resistance. Transient receptor potential (TRP) cation channels are a superfamily of non-selective $\mathrm{Ca}^{2+}$-permeable channels that regulate intracellular calcium signaling and are involved in the regulation of various characteristics of cancer cells. Emerging evidence shows a close connection between TRP channels and multiple myeloma. This review summarizes the roles of TRP channels in multiple myeloma progression, metastasis, bone destruction, and drug resistance. TRPV1 and TRPV2 orchestrate the progression of multiple myeloma, while TRPM7 promotes myeloma cell dissemination and spreading. TRPV2 and TRPV4, that activate osteoclasts, contribute to the development of osteolytic bone disease caused by multiple myeloma. Both TRPV1 inhibition and TRPV2 activation synergize with bortezomib in the chemotherapy of multiple myeloma, and TRPC1 can determine the responsiveness of multiple myeloma to MTI-101, a cyclic beta-hairpin peptide.
\end{abstract}

Correspondence to: Professor Lintao $\mathrm{Bi}$, Department of Hematology and Oncology, China-Japan Union Hospital of Jilin University, 126 XianTai Street, Changchun, Jilin 130033, P.R. China E-mail: bilt@jlu.edu.cn

*Contributed equally

Abbreviations: MM, multiple myeloma; TRP, transient receptor potential; SOCE, store-operated calcium entry; Orai1, calcium release-activated calcium modulator 1; STIM1, stromal interaction molecule 1; NFATC1, nuclear factor of activated T-cells cytoplasmic 1; RANKL, receptor activator of nuclear factor $-\kappa \mathrm{B}$ ligand; SOCs, store-operated calcium channels; DRG, dorsal root ganglia

Key words: transient receptor potential channels, calcium, multiple myeloma, disease progression, drug resistance
Antagonizing TRPA1 can alleviate bortezomib-induced painful peripheral neuropathy. Future studies in this field may identify certain TRP channels as markers or therapeutic targets for predicting the prognosis, preventing progression, and improving drug responsiveness in patients with multiple myeloma.

\section{Contents}

1. Introduction

2. TRP channels in the progression of multiple myeloma (MM)

3. TRP channels in the bone destruction of MM

4. TRP channels in the chemotherapy of MM

5. Conclusion

\section{Introduction}

Multiple myeloma (MM), although a relatively uncommon disease, is the second most commonly diagnosed hematologic malignancy after lymphoma $(1,2)$. MM, also known as plasma cell myeloma, begins with monoclonal proliferation of plasma cells in the bone marrow and then spreads throughout the body via the bloodstream, resulting in significant bone destruction and displacement of normal hematopoietic cells (3). Despite recent advances in chemotherapeutic treatment, $\mathrm{MM}$ is still an incurable disease characterized by remission and relapse in the majority of patients (4). The molecular mechanisms of many aspects of MM, including malignant transformation, progression and dissemination, bone destruction, and chemotherapy resistance, are not fully understood. Importantly, all these characteristics of MM are seemingly associated with abnormal calcium homeostasis and signaling (5). Intracellular $\mathrm{Ca}^{2+}$ signaling, as an important second messenger, regulates tumor cell behavior, and altered calcium signaling may contribute to cancer progression, metastasis, and resistance to chemotherapy (6). Calcium channels that control $\mathrm{Ca}^{2+}$ influx and remodel cellular calcium homeostasis regulate various cancer cell hallmarks such as proliferation, migration, and invasion (7). Emerging evidence indicates that calcium channels are involved in the development, progression, and chemotherapy of MM (5). 
Transient receptor potential (TRP) cation channels are a superfamily of non-selective $\mathrm{Ca}^{2+}$-permeable channels consisting of six subfamilies: TRPV (vanilloid), TRPM (melastatin), TRPA (ankyrin), TRPC (canonical), TRPP (polycystic), and TRPML (mucolipin) in mammals (8). Most TRP channels are non-selective $\mathrm{Ca}^{2+}$ permeable, while some of them are also permeable to sodium and magnesium cations. Many members in the TRPV, TRPM, and TRPA subfamilies are thermosensory channels, also known as thermoTRPs, which are activated by changes in ambient temperature ranging from noxious cold to unpleasant heat (9). Recent studies found that the S1-S4 domain in the protein structure of the TRPV1 channel significantly contributes to thermosensing and temperature-dependent activation (10). Although the thermoTRPs such as TRPV1 and TRPV2 have similar function in mediating $\mathrm{Ca}^{2+}$ entry, they have distinct functions in cellular processes by changing their expression levels and responding to different stimulants (11). We realized that these thermosensory TRP channels can also detect various endogenous stress stimuli and respond to tissue damage (12). Members in the TRPC subfamily are considered as calcium release activated channels that contribute to store-operated calcium entry (SOCE) through interacting with calcium release-activated calcium modulator 1 (Orai1) and stromal interaction molecule 1 (STIM1) (13). Although the primary roles of TRP channels are perception of a variety of sensations such as pain, temperature, tastes, pressure, and vision, accumulative evidence demonstrates that TRP channels also participate in the regulation of proliferation, differentiation, and apoptosis of malignant cells through regulating intracellular $\mathrm{Ca}^{2+}$ signaling (14-16). It has been demonstrated that changes in the expression of TRP channels can regulate tumor cell proliferation and their sensitivity to chemotherapy, suggesting roles of TRP channels in malignancy-promoting effects and resistance to antitumor chemotherapy (17). Moreover, TRP channels have been reported to be linked to overall survival and progression of various hematological malignancies, including leukemia and lymphoma (18). During recent years, there has been an increased interest in medical research into the association between TRP channels and MM, leading to the discovery of a variety of roles and functions of TRP channels in MM tumorigenesis and progression, dissemination and metastasis, MM-related osteolytic bone disease, and chemotherapy resistance (5). In this review, we summarize the evidence of the connections between TRP channels and MM (Table I).

\section{TRP channels in the progression of multiple myeloma (MM)}

MM is a plasma cell malignancy characterized by uncontrolled proliferation of plasma cell clones (19). It is broadly accepted that the tumorigenesis of MM is primarily linked to multiple genetic aberrations, including chromosome abnormalities, translocations, copy number variations, and somatic mutations, which alter several cellular processes and molecular pathways (19). These alterations that subsequently lead to neoplastic transformation and progression involve abnormal intracellular calcium signaling and altered expression of many calcium channels, including TRP channels $(5,18,20,21)$.
TRPV2 predicts the survival of patients with MM. TRPV2 is a nonspecific cation channel which is a member of the TRP vanilloid subfamily (22). TRPV2 can be activated by noxious temperatures greater than $52^{\circ} \mathrm{C}$ and plays an important role in cellular functions including cell proliferation and apoptosis $(23,24)$. Previous studies demonstrated that TRPV2 was overexpressed in MM cell lines as well as in myeloma samples from patients with MM and that TRPV2 was one of the two most significantly upregulated genes in the 17p11.2-p12 region that is an unstable chromosomal region and amplified in primary MM tumors (25). The overexpression of TRPV2 might be associated with the abnormal monoclonal proliferation of MM tumor cells (26). Bai et al (27) confirmed that TRPV2 was highly expressed in patients with MM and found that MM patients with higher transcriptional levels of TRPV2 had shorter overall survival rates than patients with lower TRPV2 expression levels, indicating that TRPV2 overexpression is associated with poor prognosis in patients with MM. These results suggest that TRPV2 may serve as a marker of MM, and TRPV2 seemingly plays a detrimental role in MM progression (Fig. 1). Then, another research group found that tumor cells from patients with MM could be divided into two subpopulations, CD138 ${ }^{+} \mathrm{TRPV}^{+}$and CD138+/TRPV2tumor cells, with distinct phenotypes, and that cannabidiol, a TRPV2 agonist, decreased proliferation of CD138 $/$ TRPV2 $^{+}$ cells (28). Moreover, activation of TRPV2 with its agonists significantly decreased MM cell viability and inhibited MM cell proliferation without affecting apoptosis likely though regulating intracellular free calcium levels $(27,29)$. These results seem to conflict with previous findings. A reasonable explanation is that overexpression of TRPV2 might serve as a compensatory response to the development and progression of MM. Nevertheless, these findings suggest that overexpression of TRPV2 may become a biomarker of MM and that TRPV2 may become a potential therapeutic target for the treatment of MM (24).

TRPVI promotes MM cell survival via acid sensation. It is well known that an acidic tumor microenvironment is a driving force promoting cancer development and progression by activating tumor cell proliferation and survival signals (30). TRPV1, a member of the TRP vanilloid subfamily, is a non-selective cation channel that serves as a sensor of noxious heat with temperature greater than $43^{\circ} \mathrm{C}$ (31). TRPV1 can also be activated by a wide variety of endogenous physical and chemical stimuli, including acidic conditions with $\mathrm{pH}$ values lower than 5.9 (31). Myeloma cells stimulate and activate osteoclasts to produce numerous protons to form an acidic tumor microenvironment, in which the $\mathrm{pH}$ values are usually below $5.5(32,33)$. It has been reported that the TRPV1 channel on MM cells is activated in the MM acidic microenvironment, which subsequently activates PI3K/Akt survival signaling and further increases the expression of TRPV1 in MM cells to enhance acid sensation (34). The PI3K/Akt pathway was found to be aberrantly activated in myeloma cells from patients with MM and to play a crucial role in survival and chemotherapy resistance of myeloma cells $(34,35)$. This positive feedback loop between upregulation of TRPV1 as an acid sensor and activation of the PI3K/Akt pathway forms a vicious cycle that could contribute to myeloma progression (Fig. 1). 
Table I. Role of TRP channels in MM.

\begin{tabular}{llc} 
TRP & \multicolumn{1}{c}{ Function and role in MM } \\
\hline TRPV1 & Promotes MM cell survival via acid sensation \\
& TRPV1 inhibition synergizes with bortezomib in chemotherapy & $(34)$ \\
TRPV2 & Upregulated in MM and is associated with poor prognosis & $(45)$ \\
& Activates osteoclasts and promotes lytic bone disease & $(25-29)$ \\
TRPV4 & Triggers osteoclastic activity \\
TRPM7 & Promotes MM metastasis and dissemination \\
TRPM8 & Highly expressed in MM & $(36)$ \\
TRPA1 & TRPA1 inhibition attenuates bortezomib-induced neuropathy \\
TRPC1 & Mediates the anti-MM effects of MTI-101 & $(42)$ \\
\hline
\end{tabular}

TRP, transient receptor potential; MM, multiple myeloma.

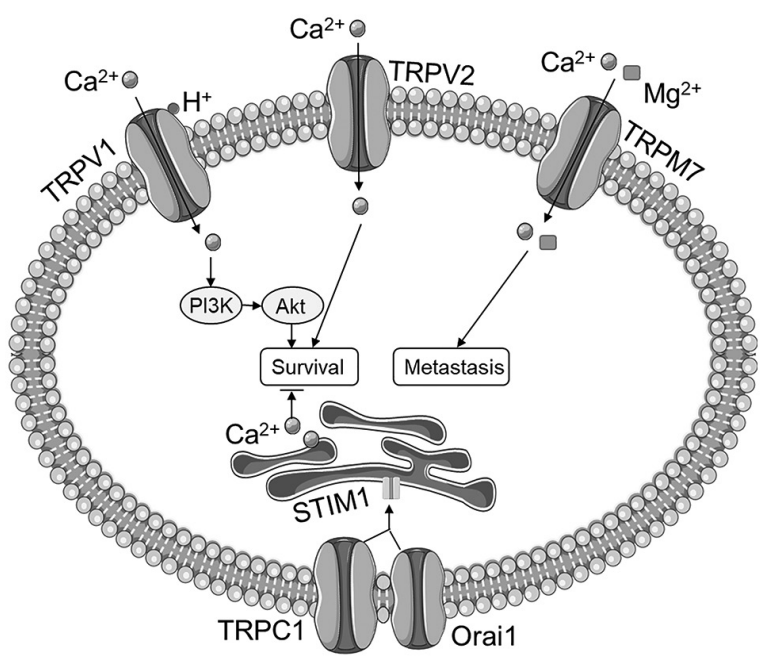

Figure 1. Role of TRP channels in the survival and metastasis of myeloma cells. Activation of TRPV1 by acidic tumor microenvironment promotes myeloma cell survival through the PI3K/Akt pathway. TRPV2 regulates myeloma cell survival and progression of multiple myeloma. TRPM7, a $\mathrm{Ca}^{2+}$ and $\mathrm{Mg}^{2+}$-permeable cation channel, promotes myeloma cell metastasis and dissemination. TRPC1 mediates calcium overload through interacting with Orai1 and STIM1, leading to myeloma cell apoptosis. TRP, transient receptor potential; Orai1, calcium release-activated calcium modulator 1; STIM1, stromal interaction molecule 1 .

TRPM7 promotes MM metastasis. MM arises from the bone marrow and then continuously spreads or disseminates to multiple sites throughout the body (36). Although the molecular mechanisms of the metastasis process are not fully understood, intracellular $\mathrm{Ca}^{2+}$ signaling is believed to play an important role in tumor cell motility and migration (37). It has been shown that TRPM7 is significantly upregulated in myeloma cells compared with normal plasma cells and that knockout or inhibition of TRPM7 inhibits MM cell migration and dissemination (38). These findings suggest that TRPM7 is a critical regulator of MM cell motility and dissemination and could become a therapeutic target for the prevention and/or treatment of metastatic MM (Fig. 1).

TRPM8 may become a marker of MM. TRPM8 serves as a receptor of cool temperature below $27^{\circ} \mathrm{C}$ and menthol and is the primary molecular transducer of non-noxious cool somatosensation in humans (39). TRPM8 is upregulated in a variety of malignant tumors such as prostate cancer $(40,41)$. Hirai et al (42) reported that TRPM8 is highly expressed in myeloma cells compared with normal plasma cells. It has also been reported that TRPM8 contributes to proliferation, survival, and invasion of cancer cells and that overexpression of TRPM8 is associated with tumor progression. However, the role of TRPM8 in the progression of MM warrants further investigation (40).

\section{TRP channels in the bone destruction of $M M$}

Osteolytic bone disease is a hallmark and a frequent complication of MM. Up to $80 \%$ of patients with MM have osteolytic bone lesions, leading to a significant cause of morbidity and mortality (43). Under physiological conditions, there is a balance between bone formation and resorption, which are mediated by osteoblastic and osteoclastic activity, respectively. Enhanced osteoclastic activity is considered as the culprit in the development of osteolytic bone lesions in patients with MM (44). Intracellular $\mathrm{Ca}^{2+}$ is an important second messenger that regulates the activity of both osteoblasts and osteoclasts.

TRPV4 triggers osteoclastic activity. Members of the TRP vanilloid subfamily, especially TRPV1, TRPV2, TRPV4, and TRPV5, have been identified to contribute to intracellular $\mathrm{Ca}^{2+}$ signaling in bone cells and bone homeostasis (45). For example, TRPV4-mediated $\mathrm{Ca}^{2+}$ influx is required for osteoclast differentiation by triggering nuclear factor of activated T-cells cytoplasmic 1 (NFATC1)-dependent gene expression (46). Moreover, knockout of TRPV4 or TRPV1 increased bone mass in a mouse model (47). Therefore, antagonizing TRPV4 may become a novel strategy to prevent or treat MM-associated bone destruction.

TRPV2 activates osteoclasts. Activation of the receptor activator of nuclear factor- $\kappa \mathrm{B}$ ligand (RANKL)/RANK signaling pathway plays a crucial role in osteoclast differentiation and activation, leading to accelerated bone resorption and lytic bone lesions (48). It has been reported that TRPV2-mediated $\mathrm{Ca}^{2+}$ influx promotes osteoclastic differentiation and activation 
by modulating calcineurin-NFAT activity and the release of RANKL and that blocking $\mathrm{Ca}^{2+}$ entry attenuates MM-induced osteoclast formation (27). These findings suggest that blocking the TRPV2 channel may provide a promising therapeutic strategy for osteolytic bone disease in patients with MM.

\section{TRP channels in the chemotherapy of MM}

Chemotherapy for MM is performed utilizing medications with different mechanisms of action, including alkylating agents, proteasome inhibitors, histone deacetylase inhibitors, and monoclonal antibodies (49-51). Although the survival outcomes of patients with MM have improved substantially due to advances in chemotherapy, myelomas frequently relapse or become refractory to chemotherapy mostly due to drug resistance, which serves as a major hurdle towards improving the long-term outcomes of patients with MM (52). Chemotherapy-induced tumor cell apoptosis and necrosis are closely regulated by cytoplasmic and mitochondrial calcium signaling (53). Evidence shows that aberrant calcium circularity and abnormal expression of calcium channels are involved in the drug resistance of MM (54).

TRPVI inhibition synergizes with bortezomib. The proteasome which degrades ubiquitylated abnormal or misfolded proteins contributes to maintenance of the immortal phenotype of myeloma cells (55). Bortezomib, a proteasome inhibitor, is used to treat MM by inducing accumulation of misfolded proteins and pro-apoptotic factors, which triggers apoptosis of myeloma cells (56). However, bortezomib-induced unfolded protein response can increase expression of chaperons such as HSP70, which restores the folding capacity, promotes cellular survival, and subsequently reduces the responsiveness and induces resistance to bortezomib (57). TRPV1 is a calcium-permeable channel expressed in the cytoplasmic and mitochondrial membrane, and activation of TRPV1 with capsaicin upregulates HSP70 (58). Beider et al (45) found that inhibition of TRPV1 with AMG9810 disturbed mitochondrial calcium signaling, abolished bortezomib-induced mitochondrial HSP70 accumulation and synergistically induced myeloma cell apoptosis with bortezomib. They also found that bortezomib plus the TRPV1 antagonist AMG9810 effectively reduced MM tumor burden in a mouse model (45). Thus, TRPV1 inhibition could be used to increase the sensitivity of bortezomib in treating MM.

TRPV2 activation synergizes with bortezomib. Similar to TRPV1, targeting TRPV2 also shows synergism with bortezomib in treating MM. Morelli et al demonstrated that activation of TRPV2 with cannabidiol synergistically inhibited myeloma cell growth and induced myeloma cell death in combination with bortezomib through suppressing extracellular-regulated kinase, serine/threonine protein kinase $\mathrm{B}$, and nuclear factor $\kappa \mathrm{B}(28)$. In addition, the therapeutic effects of cannabidiol were only present in TRPV $2^{+/+}$myeloma cells but not in TRPV2 ${ }^{-/-}$tumor cells (28). These findings suggest that TRPV2 agonists could be used to enhance the responsiveness of MM patients to bortezomib treatment.

TRPC1 determines the responsiveness to MTI-101. MTI-101, a cyclic beta-hairpin peptide, has been developed to treat patients with MM through binding to a CD44/integrin $\alpha 4 \beta 1$-containing complex and provoking myeloma cell necrosis. The mechanisms underlying the therapeutic effects of MTI-101 include inducing robust calcium influx, increasing levels of reactive oxygen species, and depolarizing the mitochondrial membrane potential, all of which are hallmarks of necrotic cell death. It has been reported that MTI-101-induced cell death is largely due to mitochondrial calcium overload through components of store-operated calcium channels (SOCs) such as TRPC1, as knockdown of TRPC1 was found to abolish MTI-101-induced necrosis (59). TRPC1 is the first identified mammalian TRP channel and is considered as a component of SOCs along with Orai1 and STIM1 (60). Treatment with MTI-101 remodels calcium homeostasis and circuitry, and resistance to MTI-101 is associated with downregulation of TRPC1 and TRPM7 and upregulation of TRPM6 and TRPM8 in MM cells (59). Further studies have demonstrated that MTI-101 induced TRPC1 translocating to the cytoplasmic membrane and forming a complex with STIM1 and that TRPC1, TRPC4, and TRPC5 were required for MTI-101-induced calcium influx and cell death (61). Importantly, the expression levels of SOC components other than TRPC channels in myelomas were identified as poor prognostic indicators in patients with MM (61). These findings suggest that the expression profile of TRPC1 may be a determinant of responsiveness to MTI-101 in MM (Fig. 1).

TRPA1 serves as a therapeutic target for bortezomib-induced peripheral neuropathy. As mentioned above, bortezomib is broadly used to treat patients with MM. In addition to drug resistance, bortezomib-induced painful peripheral neuropathy significantly limits its clinical use (62). Although the underlying mechanisms of bortezomib-induced peripheral neuropathy are not fully understood, neurophysiological abnormalities in dorsal root ganglia (DRG) and nociceptive Ad/C peripheral nerve fibers are believed to contribute to mechanical allodynia caused by bortezomib treatment (63). TRPA1, the only member in the TRPA subfamily, contains $14 \mathrm{~N}$-terminal ankyrin repeats and is expressed primarily in DRG and A $\delta$ and $\mathrm{C}$ fibers (64). Activated by cold temperature and other chemical and physical stimuli, TRPA1 functions as a mechanical and chemical stress sensor that conveys pain sensation to the brain (65). It was demonstrated that the expression level of TRPA1 was significantly increased in DRG of rats treated with bortezomib, suggesting that this upregulation might contribute to bortezomib-induced peripheral neuropathy and neuropathic pain (66). Moreover, blocking TRPA1 ameliorated bortezomib-elicited mechanical pain and cold sensitivity in rats (66). Further study demonstrated that interleukin-6 (IL-6) was responsible for bortezomib-induced TRPA1 upregulation and that blocking the IL- 6 signaling reduced TRPA1 expression and attenuated mechanical hyperalgesia and cold hypersensitivity in rats treated with bortezomib (67). These findings suggest that TRPA1 may become a therapeutic target for bortezomib-induced painful peripheral neuropathy in patients with MM.

\section{Conclusion}

TRP channels connect tumor microenvironment stimuli to intracellular calcium signaling and play crucial roles in various 
behaviors of cancer cells. In this review, we summarize the currently available evidence showing the roles of TRP channels in many aspects of MM, including tumor progression, dissemination, bone destruction, and drug resistance. This is an emerging field, and there are still a lot of unknown questions regarding the role of TRP channels in the development and progression of MM. It is challenging to treat MM by targeting TRP channels. As discussed, activation of TRPV2 was found to improve the sensitivity of myeloma cells to chemotherapy but may exacerbate MM-related bone disease by stimulating osteoclasts. Therefore, selectively targeting TRP channels in myeloma cells or osteoclasts may be required to delivery optimal treatment. Many previous studies in this topic were performed in cultured cells and animal models; therefore, the clinical significance of the role of TRP channels in MM is not well documented. Especially, the role of TRPV1, TRPV2, TRPV4, TRPC1, and TRPM7 in MM should be evaluated in the clinical setting. Future studies may identify certain TRP channels as markers or therapeutic targets for predicting prognosis, preventing disease progression, and improving drug responsiveness of MM. Further understanding of the role of TRP channels in MM may benefit the survival and long-term outcome of patients with MM.

\section{Acknowledgements}

Not applicable.

\section{Funding}

This work was supported by grants from the National Key R\&D Program of China (2018YFC0116901 and 2018YFC1315604), the Science and Technology Development Project of Jilin Province (20180101124JC), the Special Project for Health Research of Jilin Province (2018SCZ031 and 2019SCZ055), and the Health Technology Innovation Project of Jilin Province (3D517ED43430).

\section{Availability of data and materials}

Data sharing is not applicable to this article, as no datasets were generated or analyzed during the current study.

\section{Authors' contributions}

Conceptualization of the review concept and manuscript drafting were conducted by LM. Literature review and manuscript revision were carried out by GG. Conceptualization of the review concept, supervision, and manuscript revision were conducted by LB. Data authentication is not applicable. All authors read and approved the final version of the manuscript for publication.

\section{Ethics approval and consent to participate}

Not applicable.

\section{Patient consent for publication}

Not applicable.

\section{Competing interests}

The authors declare that they have no competing interests.

\section{References}

1. van de Donk N, Pawlyn C and Yong KL: Multiple myeloma. Lancet 397: 410-427, 2021.

2. Ghandili S, Weisel KC, Bokemeyer C and Leypoldt LB: Current treatment approaches to newly diagnosed multiple myeloma. Oncol Res Treat 44: 690-699, 2021.

3. Kazandjian D: Multiple myeloma epidemiology and survival: A unique malignancy. Semin Oncol 43: 676-681, 2016.

4. Costa LJ and Usmani SZ: Defining and managing high-risk multiple myeloma: Current concepts. J Natl Compr Canc Netw 18: 1730-1737, 2020.

5. Li T, Chen J and Zeng Z: Pathophysiological role of calcium channels and transporters in the multiple myeloma. Cell Commun Signal 19: 99, 2021.

6. Stewart TA, Yapa KT and Monteith GR: Altered calcium signaling in cancer cells. Biochim Biophys Acta 1848: 2502-2511, 2015.

7. Deliot $\mathrm{N}$ and Constantin B: Plasma membrane calcium channels in cancer: Alterations and consequences for cell proliferation and migration. Biochim Biophys Acta 1848: 2512-2522, 2015.

8. Nilius B, Owsianik G, Voets T and Peters JA: Transient receptor potential cation channels in disease. Physiol Rev 87: 165-217, 2007.

9. Ferrandiz-Huertas C, Mathivanan S, Wolf CJ, Devesa I and Ferrer-Montiel A: Trafficking of ThermoTRP channels. Membranes (Basel) 4: 525-564, 2014.

10. Kim M, Sisco NJ, Hilton JK, Montano CM, Castro MA, Cherry BR, Levitus M and Horn WD: Evidence that the TRPV1 S1-S4 membrane domain contributes to thermosensing. Nat Commun 11: 4169, 2020.

11. Gorbunov AS, Maslov LN, Jaggi AS, Singh N, Petrocellis LD, Boshchenko AA, Roohbakhsh A, Bezuglov VV and Oeltgen PR: Physiological and pathological role of TRPV1, TRPV2 and TRPV4 channels in heart. Curr Cardiol Rev 15: 244-251, 2019.

12. Vay L, Gu C and McNaughton PA: The thermo-TRP ion channel family: Properties and therapeutic implications. $\mathrm{Br}$ J Pharmacol 165: 787-801, 2012.

13. Ambudkar IS, de Souza LB and Ong HL: TRPC1, orai1, and STIM1 in SOCE: Friends in tight spaces. Cell Calcium 63: 33-39, 2017.

14. Santoni G and Farfariello V: TRP channels and cancer: new targets for diagnosis and chemotherapy. Endocr Metab Immune Disord Drug Targets 11: 54-67, 2011.

15. Canales J, Morales D, Blanco C, Rivas J, Díaz N, Angelopoulos I and Cerda O: A TR(i)P to cell migration: New roles of TRP channels in mechanotransduction and cancer. Front Physiol 10: 757, 2019.

16. Prevarskaya N, Zhang L and Barritt G: TRP channels in cancer. Biochim Biophys Acta 1772: 937-946, 2007.

17. Santoni G, Maggi F, Morelli MB, Santoni M and Marinelli O: Transient receptor potential cation channels in cancer therapy. Med Sci (Basel) 7: 108, 2019.

18. Maggi F, Morelli MB, Nabissi M, Marinelli O, Zeppa L, Aguzzi C, Santoni G and Amantini C: Transient receptor potential (TRP) channels in haematological malignancies: An update. Biomolecules 11: 765, 2021.

19. Heider M, Nickel K, Hogner M and Bassermann F: Multiple myeloma: Molecular pathogenesis and disease evolution. Oncol Res Treat 44: 672-681, 2021.

20. Hodeify R, Yu F, Courjaret R, Nader N, Dib M, Sun L, Adap E, Hubrack S and Machaca K: Regulation and Role of Store-Operated $\mathrm{Ca}(2+)$ Entry in Cellular Proliferation. In: Calcium Entry Channels in Non-Excitable Cells. Kozak JA and Putney JW Jr (eds). CRC Press/Taylor \& Francis, Boca Raton, FL, pp215-240, 2018.

21. Karki T and Tojkander S: TRPV protein family-from mechanosensing to cancer invasion. Biomolecules 11: 1019, 2021.

22. Kojima I and Nagasawa M: Trpv2. Handb Exp Pharmacol 222: 247-272, 2014.

23. Liberati S, Morelli MB, Amantini C, Santoni M, Nabissi M, Cardinali $\mathrm{C}$ and Santoni G: Advances in transient receptor potential vanilloid-2 channel expression and function in tumor growth and progression. Curr Protein Pept Sci 15: 732-737, 2014. 
24. Siveen KS, Nizamuddin PB, Uddin S, Al-Thani M, Frenneaux MP, Janahi IA, Steinhoff M and Azizi F: TRPV2: A cancer biomarker and potential therapeutic target. Dis Markers 2020: 8892312, 2020.

25. Fabris S, Todoerti K, Mosca L, Agnelli L, Intini D, Lionetti M, Guerneri S, Lambertenghi-Deliliers G, Bertoni F and Neri A: Molecular and transcriptional characterization of the nove 17p11.2-p12 amplicon in multiple myeloma. Genes Chromosomes Cancer 46: 1109-1118, 2007.

26. Liberati S, Morelli MB, Amantini C, Farfariello V, Santoni M, Conti A, Nabissi M, Cascinu S and Santoni G: Loss of TRPV2 homeostatic control of cell proliferation drives tumor progression. Cells 3: 112-128, 2014.

27. Bai H, Zhu H, Yan Q, Shen X, Lu X, Wang J, Li J and Chen L: TRPV2-induced $\mathrm{Ca}(2+)$-calcineurin-NFAT signaling regulates differentiation of osteoclast in multiple myeloma. Cell Commun Signal 16: 68, 2018.

28. Morelli MB, Offidani M, Alesiani F, Discepoli G, Liberati S, Olivieri A, Santoni M, Santoni G, Leoni P and Nabissi M: The effects of cannabidiol and its synergism with bortezomib in multiple myeloma cell lines. A role for transient receptor potential vanilloid type-2. Int J Cancer 134: 2534-2546, 2014

29. Nabissi M, Offidani M, Morelli MB, Pettinari M, Caraffa P, Gentili S, Corvatta L, Santoni G, Alesiani F and Leoni P: TRPV2 expression and its role in proliferation of human multiple myeloma cell lines. Blood 118: 5003, 2011.

30. Boedtkjer E and Pedersen SF: The acidic tumor microenvironment as a driver of cancer. Annu Rev Physiol 82: 103-126, 2020.

31. Caterina MJ, Schumacher MA, Tominaga M, Rosen TA Levine JD and Julius D: The capsaicin receptor: A heat-activated ion channel in the pain pathway. Nature 389: 816-824, 1997.

32. Swietach P, Vaughan-Jones RD, Harris AL and Hulikova A: The chemistry, physiology and pathology of $\mathrm{pH}$ in cancer. Philos Trans R Soc Lond B Biol Sci 369: 20130099, 2014.

33. Gastelum G, Veena M, Lyons K, Lamb C, Jacobs N, Yamada A, Baibussinov A, Sarafyan M, Shamis R, Kraut J and Frost P: Can targeting hypoxia-mediated acidification of the bone marrow microenvironment kill myeloma tumor cells? Front Oncol 11: 703878, 2021.

34. Amachi R, Hiasa M, Teramachi J, Harada T, Oda A, Nakamura S, Hanson D, Watanabe K, Fujii S, Miki H, et al: A vicious cycle between acid sensing and survival signaling in myeloma cells: acid-induced epigenetic alteration. Oncotarget 7: 70447-70461, 2016.

35. Ramakrishnan V and Kumar S: PI3K/AKT/mTOR pathway in multiple myeloma: From basic biology to clinical promise. Leuk Lymphoma 59: 2524-2534, 2018.

36. Ghobrial IM: Myeloma as a model for the process of metastasis: Implications for therapy. Blood 120: 20-30, 2012.

37. White $C$ : The regulation of tumor cell invasion and metastasis by endoplasmic reticulum-to-mitochondrial $\mathrm{Ca}(2+)$ transfer. Front Oncol 7: 171, 2017

38. Samart $\mathrm{P}$, Luanpitpong S, Rojanasakul $\mathrm{Y}$ and Issaragrisil S: O-GlcNAcylation homeostasis controlled by calcium influx channels regulates multiple myeloma dissemination. J Exp Clin Cancer Res 40: 100, 2021.

39. Pina R, Ugarte G, Campos M, Íñigo-Portugués A, Olivares E, Orio P, Belmonte C, Bacigalupo J and Madrid R: Role of TRPM8 channels in altered cold sensitivity of corneal primary sensory neurons induced by axonal damage. J Neurosci 39: 8177-8192, 2019.

40. Yee NS: Roles of TRPM8 ion channels in cancer: Proliferation, survival, and invasion. Cancers (Basel) 7: 2134-2146, 2015.

41. Bidaux G, Flourakis M, Thebault S, Zholos A, Beck B, Gkika D, Roudbaraki M, Bonnal JL, Mauroy B, Shuba Y, et al: Prostate cell differentiation status determines transient receptor potential melastatin member 8 channel subcellular localization and function. J Clin Invest 117: 1647-1657, 2007.

42. Hirai A, Aung NY, Ohe R, Nishida A, Kato T, Meng H, Ishizawa K, Fujii J and Yamakawa M: Expression of TRPM8 in human reactive lymphoid tissues and mature B-cell neoplasms. Oncol Lett 16: 5930-5938, 2018

43. Terpos E, Morgan G, Dimopoulos MA, Drake MT, Lentzsch S, Raje N, Sezer O, García-Sanz R, Shimizu K, Turesson I, et al: International myeloma working group recommendations for the treatment of multiple myeloma-related bone disease. J Clin Oncol 31: 2347-2357, 2013 .

44. Terpos E, Ntanasis-Stathopoulos I, Gavriatopoulou M and Dimopoulos MA: Pathogenesis of bone disease in multiple myeloma: From bench to bedside. Blood Cancer J 8: 7, 2018.
45. Beider K, Rosenberg E, Dimenshtein-Voevoda V, Sirovsky Y, Vladimirsky J, Magen H, Ostrovsky O, Shimoni A, Bromberg Z, Weiss L, et al: Blocking of transient receptor potential vanilloid 1 (TRPV1) promotes terminal mitophagy in multiple myeloma, disturbing calcium homeostasis and targeting ubiquitin pathway and bortezomib-induced unfolded protein response. J Hematol Oncol 13: 158, 2020

46. Masuyama R, Vriens J, Voets T, Karashima Y, Owsianik G, Vennekens R, Lieben L, Torrekens S, Moermans K, Bosch AV, et al: TRPV4-mediated calcium influx regulates terminal differentiation of osteoclasts. Cell Metab 8: 257-265, 2008.

47. Nishimura H, Kawasaki M, Tsukamoto M, Menuki K, Suzuki H, Matsuura T, Baba K, Motojima Y, Fujitani T, Ohnishi H, et al: Transient receptor potential vanilloid 1 and 4 double knockout leads to increased bone mass in mice. Bone Rep 12: 100268 , 2020.

48. Raje NS, Bhatta S and Terpos E: Role of the RANK/RANKL pathway in multiple myeloma. Clin Cancer Res 25: 12-20, 2019.

49. Pinto V, Bergantim R, Caires HR, Seca H, Guimarães JE and Vasconcelos MH: Multiple myeloma: Available therapies and causes of drug resistance. Cancers (Basel) 12: 407, 2020.

50. Painuly U, Ramakrishnan V, Kimlinger T, Wellik L, Haug J, Gonsalves W, Bi L, Huang Z, Rajkumar SV and Kumar S: Aurora kinase and FGFR3 inhibition results in significant apoptosis in molecular subgroups of multiple myeloma. Oncotarget 9: 34582-34594, 2018.

51. Ramakrishnan V, Gomez M, Prasad V, Kimlinger T, Painuly U, Mukhopadhyay B, Haug J, Bi L, Rajkumar SV and Kumar S: Smac mimetic LCL161 overcomes protective ER stress induced by obatoclax, synergistically causing cell death in multiple myeloma. Oncotarget 7: 56253-56265, 2016.

52. Robak P, Drozdz I, Szemraj J and Robak T: Drug resistance in multiple myeloma. Cancer Treat Rev 70: 199-208, 2018.

53. Kerkhofs M, Bittremieux M, Morciano G, Giorgi C, Pinton P, Parys JB and Bultynck G: Emerging molecular mechanisms in chemotherapy: $\mathrm{Ca}(2+)$ signaling at the mitochondria-associated endoplasmic reticulum membranes. Cell Death Dis 9: 334, 2018.

54. Liu M, Wang Y, Miettinen JJ, Kumari R, Majumder MM, Tierney C, Bazou D, Parsons A, Suvela M, Lievonen J, et al: S100 calcium binding protein family members associate with poor patient outcome and response to proteasome inhibition in multiple myeloma. Front Cell Dev Biol 9: 723016, 2021.

55. Ito S: Proteasome inhibitors for the treatment of multiple myeloma. Cancers (Basel) 12: 265, 2020.

56. Robak P, Szemraj J, Mikulski D, Drozdz I, Juszczak K, Jarych D, Misiewicz M, Kościelny K, Fendler W and Robak T: Prognostic value of resistance proteins in plasma cells from multiple myeloma patients treated with bortezomib-based regimens. J Clin Med 10: 5028, 2021.

57. Zaal EA, Wu W, Jansen G, Zweegman S, Cloos J and Berkers CR: Bortezomib resistance in multiple myeloma is associated with increased serine synthesis. Cancer Metab 5: 7, 2017.

58. Bromberg Z, Goloubinoff P, Saidi Y and Weiss YG: The membrane-associated transient receptor potential vanilloid channel is the central heat shock receptor controlling the cellular heat shock response in epithelial cells. PLoS One 8: e57149, 2013

59. Emmons MF, Anreddy N, Cuevas J, Steinberger K, Yang S, McLaughlin M, Silva A and Hazlehurst LA: MTI-101 treatment inducing activation of Stim1 and TRPC1 expression is a determinant of response in multiple myeloma. Sci Rep 7: 2685, 2017.

60. Xu SZ and Beech DJ: TrpC1 is a membrane-spanning subunit of store-operated $\mathrm{Ca}(2+)$ channels in native vascular smooth muscle cells. Circ Res 88: 84-87, 2001.

61. Elzamzamy OM, Johnson BE, Chen WC, Hu G, Penner R and Hazlehurst LA: Transient receptor potential C $1 / 4 / 5$ is a determinant of MTI-101 induced calcium influx and cell death in multiple myeloma. Cells 10: 1490, 2021

62. Carozzi VA, Renn CL, Bardini M, Fazio G, Chiorazzi A, Meregalli C, Oggioni N, Shanks K, Quartu M, Serra MP, et al: Bortezomib-induced painful peripheral neuropathy: An electrophysiological, behavioral, morphological and mechanistic study in the mouse. PLoS One 8: e72995, 2013.

63. Argyriou AA, Iconomou G and Kalofonos HP: Bortezomib-induced peripheral neuropathy in multiple myeloma: A comprehensive review of the literature. Blood 112: 1593-1599, 2008. 
64. Hu F, Song X and Long D: Transient receptor potential ankyrin 1 and calcium: Interactions and association with disease (Review). Exp Ther Med 22: 1462, 2021.

65. Nilius B, Appendino G and Owsianik G: The transient receptor potential channel TRPA1: From gene to pathophysiology. Pflugers Arch 464: 425-458, 2012.
66. Wang Q, Wang J, Gao D and Li J: Inhibition of PAR2 and TRPA 1 signals alleviates neuropathic pain evoked by chemotherapeutic bortezomib. J Biol Regul Homeost Agents 31: 977-983, 2017.

67. Liu D, Sun M, Xu D, Ma X, Gao D and Yu H: Inhibition of TRPA1 and IL-6 signal alleviates neuropathic pain following chemotherapeutic bortezomib. Physiol Res 68: 845-855, 2019. 